\title{
Edge-emitting quantum well laser with integrated intracavity electrostatic gate
}

Reginald K. Lee, Yuan Jian Xu, John D. O'Brien, Oskar J. Painter, Axel Scherer, et al.

Reginald K. Lee, Yuan Jian Xu, John D. O'Brien, Oskar J. Painter, Axel Scherer, Amnon Yariv, "Edge-emitting quantum well laser with integrated intracavity electrostatic gate," Proc. SPIE 3290, Optoelectronic Integrated Circuits II, (22 December 1997); doi: 10.1117/12.298238 Jose, CA, United States 


\title{
Edge-emitting quantum well laser with integrated intracavity electrostatic gate
}

\author{
R.K. Lee, Y.J. Xu ${ }^{a}$, J. O'Brien ${ }^{b}$, O.J. Painter, A. Scherer and A. Yariv \\ Applied Physics and Electrical Engineering \\ California Institute of Technology, MS 128-95 \\ Pasadena, CA 91125 USA \\ ${ }^{b}$ Electrical Engineering / Electrophysics \\ University of Southern California, mc-0271 \\ Los Angeles, CA 90089 USA
}

\begin{abstract}
Ridge waveguide, edge-emitting single quantum well GaAs lasers with an integrated gating electrode have been fabricated. These devices integrate a MESFET structure with the laser PN junction so that the SBD (Schottky barrier diode) depletion layer can be used for transverse current confinement in the laser. Device fabrication was very simple requiring only an anisotropic etch for waveguide definition followed by a single self-aligned contact deposition step. The Schottky barrier depletion layers on either side of the ridge waveguide act to confine free carriers. This structure allows for separation of the optical and electrical confinement in the transverse direction without requiring complex fabrication. The device demonstrated modulation of the pulsed lasing threshold with gate control voltage on a 30 micron wide ridge. Above threshold, increasing power output with increasing gate voltage was demonstrated with negligible gate current. The multimode lasing spectrum showed that the increased power output occurred for all modes with no shift in the mode wavelengths to within the resolution of the measurement system.
\end{abstract}

Keywords: semiconductor laser, Schottky barrier diode, electrostatic current confinement, MESFET

\section{INTRODUCTION}

High performance index-guided lasers with transverse carrier confinement are well established, ${ }^{1-3}$ but require relatively complex processing and do not provide for separate optical and electronic confinement in the transverse direction. Separate confinement in the transverse direction is expected to further improve laser performance. One method for counteracting carrier diffusion in the lateral direction is by electrostatic repulsion using a gate which confines carriers to the center of the laser stripe. This is accomplished in practice through the addition of self-aligned gates next to the laser stripes which control the lateral distribution of electrons or holes and thereby control the threshold current. Thus, it is possible to obtain a separate carrier confinement geometry in which the optical cavity and the carrier injection geometry in the laser can both be independently optimized. The incorporation of a third control-gate electrode into a semiconductor laser, a prospect made practical by the small transverse dimensions, opens up new possibilities for controlling and modulating semiconductor lasers.

Optimization of such a device provides the opportunity to develop ultra-low threshold current devices with electrostatic control over the transverse mode pattern and light intensity. Reductions of threshold currents in such ultra-low threshold lasers have traditionally been accomplished by reducing the active gain volume while maintaining a separate confinement for the optical field. This type of structure is readily achieved in the direction of crystal growth using modern crystal growth techniques. However, achieving similar confinement in the transverse direction has been much more difficult, requiring high-resolution fabrication and material re-growth to form transverse heterostructures. In this paper we demonstrate the use of electrostatic depletion to confine carriers in a ridge waveguide semiconductor

Send correspondence to R.K.Lee

R.K.L.: Email: leereg@cco.caltech.edu; Telephone: 626-395-4829; Fax: 626-405-0928

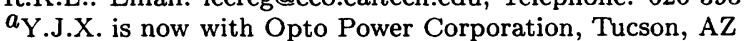




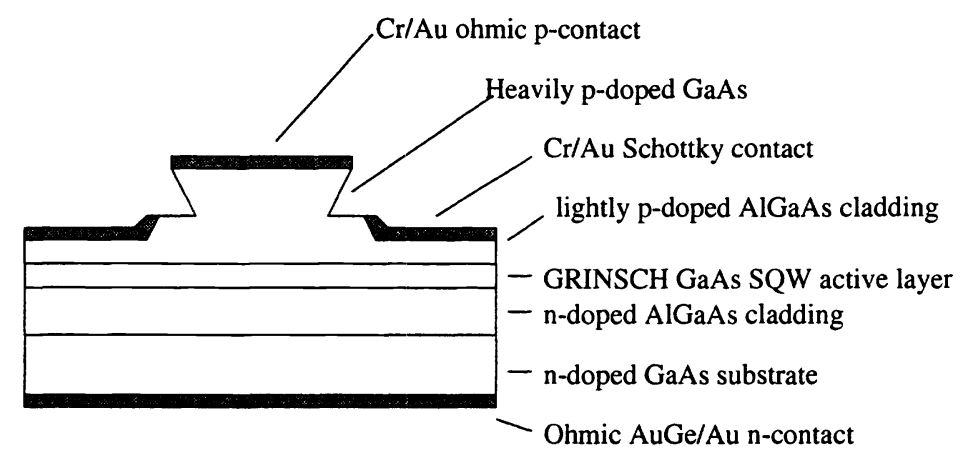

Figure 1. Cross-section of the laser structure with the extra contacts for electrostatic depletion. Schottky contacts are made to a lightly doped (background doping) layer.

laser. The experimental results presented here are for edge-emitting lasers but the technique is applicable to vertical cavity surface emitting lasers (VCSELs) as well.

To demonstrate electrostatic control over the optical laser emission through reverse-biased Schottky diodes, we have conducted preliminary tests on edge-emitting laser diodes with self-aligned gates deposited on both sides of the laser stripe. In these devices, the threshold current and output power can be adjusted by the application of an external gate voltage. As we describe in greater detail below, these preliminary data clearly show that it is possible to use reverse-biased Schottky diodes to control the performance of edge-emitters. Although only modest changes in the threshold currents of our present lasers have been achieved so far, we believe that careful optimization of both growth design and laser geometry will result in much more substantial electrostatic control over threshold currents of laser diodes. In addition to obtaining very low threshold currents, the approach taken here will also result in electrostatic control over the transverse lasing modes within the laser cavity by regulating the transverse gain distribution. This should allow the transverse beam width to be electrostatically adjusted to, for example, optimally couple the light output into an optical fiber, or to reduce the noise associated with changes in mode patterns as the driving current for the laser diode is changed.

\section{LASER DESIGN AND FABRICATION}

Our device consists of a PIN diode with contacts made to the $\mathrm{p}$ and $\mathrm{n}$ regions to bias the laser diode. In addition an electrical contact is made to the intrinsic layer between the active region and the $\mathrm{p}$ (or $\mathrm{n}$ ) side or to a low doped region near the intrinsic layer, outside the junction depletion width. This contact is used to form a depletion region to electrostatically confine the carriers. Figure 1 shows a schematic cross section of the laser structure. The electrostatic field from the depletion contacts acts to confine free carriers which are injected from the top ohmic contact, towards the center of the ridge waveguide as shown schematically in figure 2 . This depletion region can be created using a reverse-biased Schottky contact or a MOS capacitor. This contact effectively forms a field effect transistor (FET) inside the laser cavity. This is illustrated in figure 2.

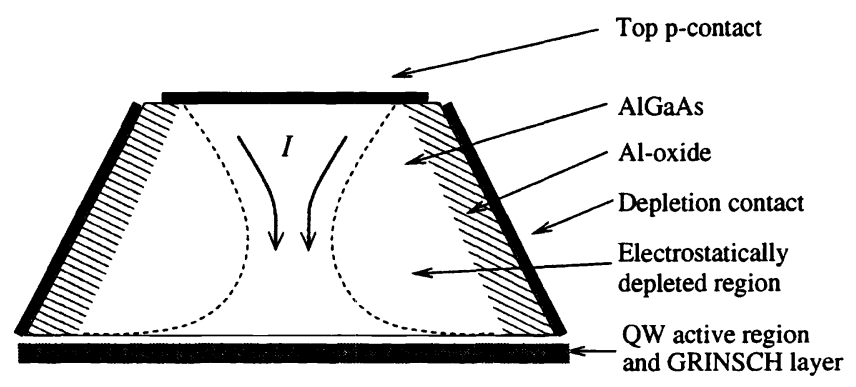

Figure 2. Illustration of current confinement through electrostatic depletion. 

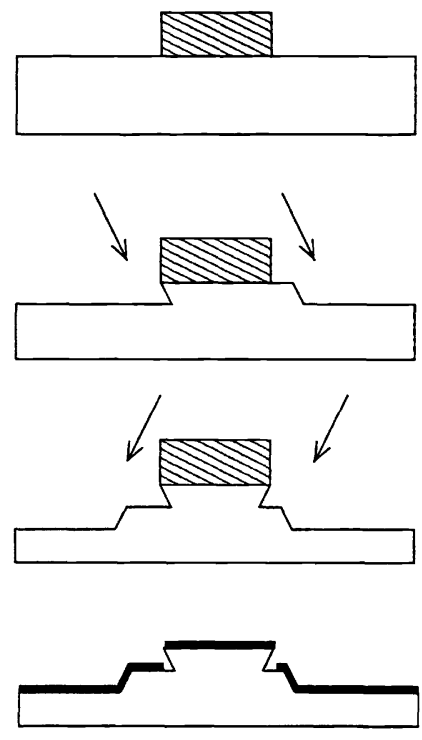

Figure 3. Process flow for ridge waveguide definition
Self-aligned metallization

of $\mathrm{Cr} / \mathrm{Au}$ contacts

Lithography of photoresist stripe mask

\author{
CAIBE etch at $35^{\circ}$ \\ from one side \\ Rotate and etch at $35^{\circ}$ \\ from other side
}

The electrical contact added to the traditional laser structure may be used to reduce the threshold current or to modulate the intensity of the laser above threshold. The threshold current of the laser can be reduced by confining the carriers to, and thereby pumping, a smaller volume. This contact scheme can be used for edge-emitting lasers as well as vertical cavity surface emitting lasers (VCSELs). Above threshold, this mechanism can be used to modulate the intensity with a constant bias current. This occurs because by electrostatically modulating the confinement volume with the same pumping current, the current density is modulated and the output intensity is also modulated. For small changes in the pumping area, as a first approximation, changes to the optical mode - gain coupling (transverse confinement factor) can be assumed to be negligible.

The laser structure was MBE-grown on a silicon doped (n-type) GaAs substrate using a solid source Riber 2300 system. The active layer consisted of a single $10 \mathrm{~nm}$ GaAs quantum well within a graded index separate confinement heterostructure (GRINSCH) with barriers graded between $A l_{0.5} G a_{0.5} A s$ and $A l_{0.2} G a_{0.8} A s$. This was surrounded on either side by cladding layers of $A l_{0.5} \mathrm{Ga}_{0.5} A s$. Doping in the cladding layers was $3 \times 10^{18} \mathrm{~cm}^{-3}$ for both $\mathrm{Si}$ and $\mathrm{Be}$ on either side of the GRINSCH region. In the upper $2 \mu \mathrm{m}$ of the p-cladding region, the doping concentration was reduced to background levels of roughly $5 \times 10^{14} \mathrm{~cm}^{-3}$, mostly due to carbon in the growth chamber. This was done in order to provide a low doped region for the Schottky contact. Above this low doped region, $1 / 3 \mu m$ of $A l_{0.5} G a_{0.5} A s$ was grown where the doping was linearly graded up to $3 \times 10^{18} \mathrm{~cm}^{-3}$. This was then capped with a $1 / 3 \mu \mathrm{m}$ GaAs contact layer with Be doping graded from $3 \times 10^{18} \mathrm{~cm}^{-3}$ to approximately $1 \times 10^{19} \mathrm{~cm}^{-3}$ so as to provide a good ohmic contact.

The ridge waveguide was defined using a $\mathrm{Cl}_{2} / \mathrm{Ar}^{+}$chemically assisted ion beam etch (CAIBE). This anisotropic etching was performed at approximately 35 degrees from the surface normal from either side of a photoresist stripe mask until the low-doped cladding region was exposed. The undercutting of the photoresist mask allows for the self-aligned deposition of $\mathrm{Cr} / \mathrm{Au}$ contacts to form both an ohmic contact to the heavily doped top of the waveguide, and Schottky contacts on the exposed low-doped cladding regions on either side of the waveguide ridge. The process flow for ridge definition is shown in figure 3 and a low angle cross-section SEM image of a completed device is shown in figure 4. The device fabrication was superficially similar to the Schottky restricted laser demonstrated by Amann ${ }^{4}$ and also by Temkin et al. ${ }^{5}$ where Schottky barriers were used to define the pumping region instead of using an oxide isolation layer. In that work, a single electrode was deposited with ohmic and Schottky regions and the Schottky barrier was used simply as a non-conductive region so as to define the current injection area. A key difference in this electrostatically controlled laser, however, lies in the use of electrostatics to confine free carriers and the separate control over the pumping and biasing electrodes by separating ohmic and Schottky contact regions. The etch for the waveguide ridge can also be performed using an anisotropic wet etch to provide the necessary undercut as shown in 
figure 5. This was done using a $1: 8: 40$ solution of $\mathrm{H}_{2} \mathrm{SO}_{4}: \mathrm{H}_{2} \mathrm{O}_{2}: \mathrm{H}_{2} \mathrm{O}$. The waveguide ridge was undercut sur that the same self-aligned contact metalization process as with the dry etched process was possible.

Once the laser stripes and biasing gates were defined, argon ion milling was used with a photoresist mask to electrically isolate the devices. Finally, the wafer was lapped down to approximately 6 mils and an AuGe/ $\mathrm{Ni} / \mathrm{Au}$ contact was applied on the n-side substrate. Reverse biasing the Schottky barrier contacts on either side of the ridge relative to the ohmic p-contact, creates depleted regions which confine the carriers towards the center of the laser ridge.

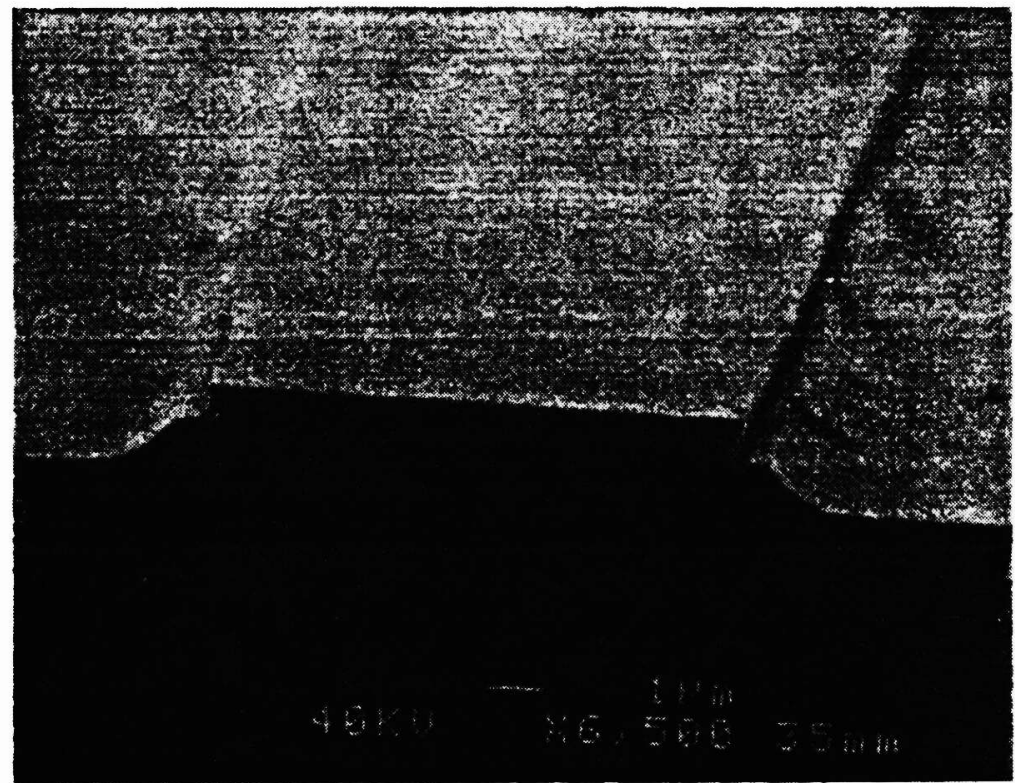

Figure 4. Low angle SEM micrograph of a completed $10 \mu m$ wide laser device in cross section

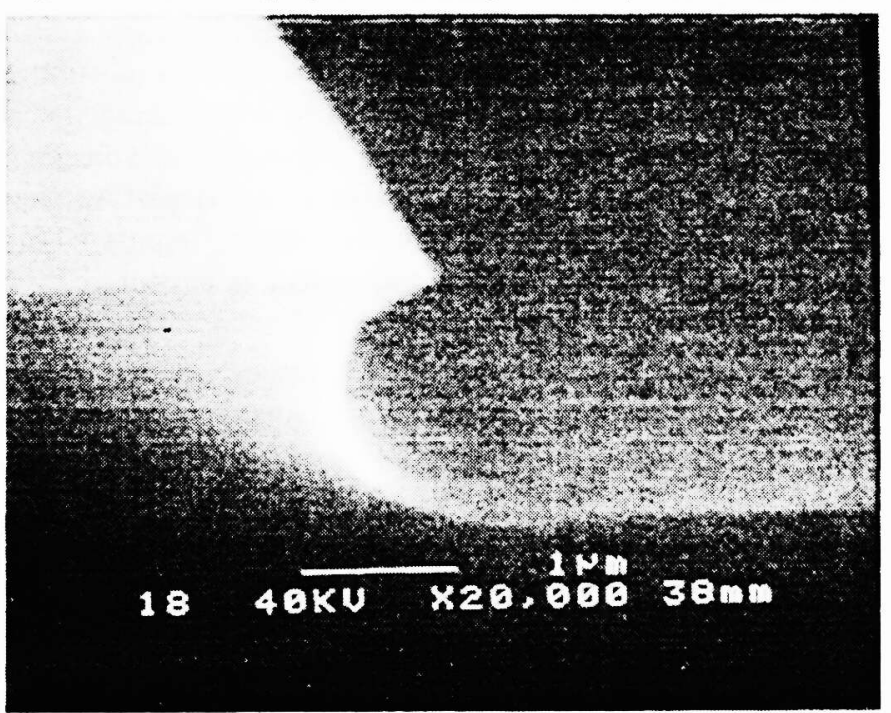

Figure 5. Waveguide undercut using $\mathrm{H}_{2} \mathrm{SO}_{4}: \mathrm{H}_{2} \mathrm{O}_{2}: \mathrm{H}_{2} \mathrm{O}$ anisotropic wet etch 


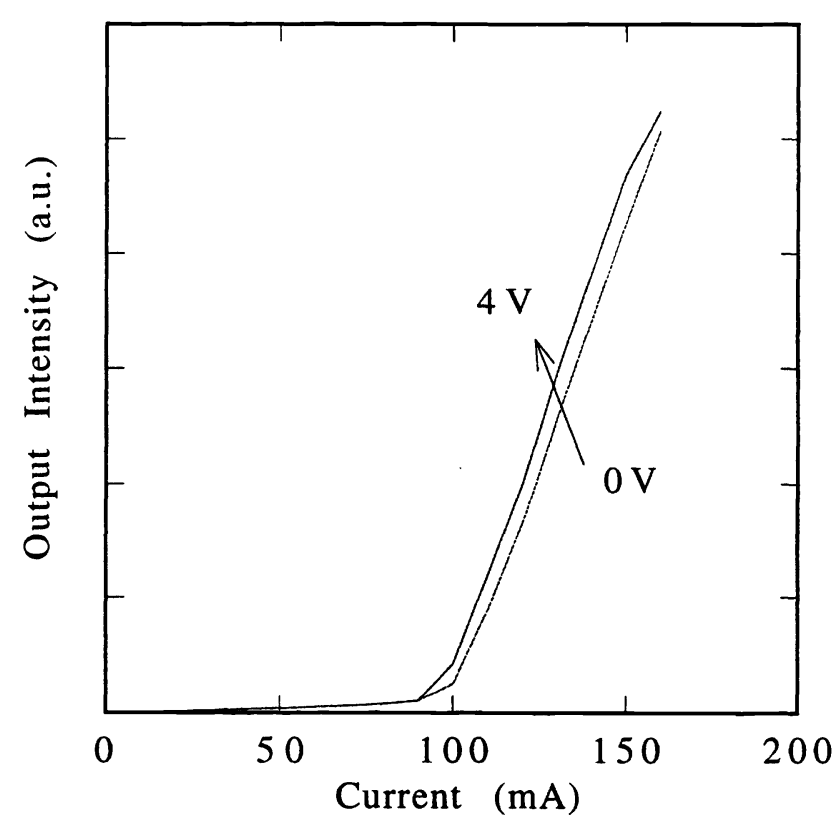

Figure 6. L-I curves for $13 \mu m$ lasers at different gate voltages showing roughly $4 \%-5 \%$ change in threshold current

\section{RESULTS AND DISCUSSION}

The light output versus pumping current curves for a $13 \mu m$ wide device at different gate voltages is shown in figure 6 . The laser was pumped with $250 \mathrm{~ns}$ pulses at $30 \mathrm{kHz}$. There is an approximately $4 \%-5 \%$ reduction in the lasing threshold current with increased gate voltage. If we assume that the threshold current density is constant for small changes in the depletion voltage, then the change in threshold should be proportional to the change in the effective active width. Thus, from the threshold change, an effective width can be calculated which should be equal to the ridge width minus the thickness of the depleted region. We have demonstrated a decrease in threshold current in a $13 \mu \mathrm{m}$ wide ridge-waveguide edge-emitting laser structure for a depletion voltage of $4 \mathrm{~V}$. Under the assumption of constant threshold current density for the laser, this translates into a depletion change of roughly $0.5 \mu m-0.6 \mu m$. Since this was a unoptimized laser structure, bigger reductions are anticipated. In this case, the gate voltage was only applied from one side of the ridge so a doubling of this effect is expected by simply applying bias from both sides.

The leakage current through the reverse biased Schottky contacts was also measured and is shown in figure 7 . It is seen that the contribution to the pumping current from leakage through the Schottky contacts is negligibly small and could not account for the increase in output power. In fact, low leakage was observed at biases much greater than the expected reverse breakdown for such Schottky contacts (figure 7). This can be expected since the Schottky contacts are deposited directly after the CAIBE etch, and onto the $A l_{0.5} G a_{0.5} A s$ surface, which may have been partially oxidized. Schottky contacts with larger reverse bias leakage currents were obtained when the samples were immersed into a dilute HF solution before contact deposition. This observation leads us to believe that a thin oxide layer, or the ion damage resulting from the CAIBE process contribute to a thin insulating layer which is formed between the gate metal and the semiconductor, resulting in a MIS-like structure. Such contacts would also account for the large measured breakdown voltages of our gate electrodes on some devices (see figure 7). Larger leakage currents were also seen when devices were made using an anisotropic wet etch to define the waveguide ridges (see figure 5). However, low leakage contacts with high breakdown voltages were achievable using the wet etching process by introducing an oxide layer prior to contact metalization through the use of steam oxidation of the AlGaAs layer to produce an aluminum-based native oxide. ${ }^{6}$ This, in effect, produces an intracavity metal-oxide semiconductor field effect transistor (MOSFET). AlGaAs based MOSFETs have previously been demonstrated using this wet oxidation technique. ${ }^{7}$ 


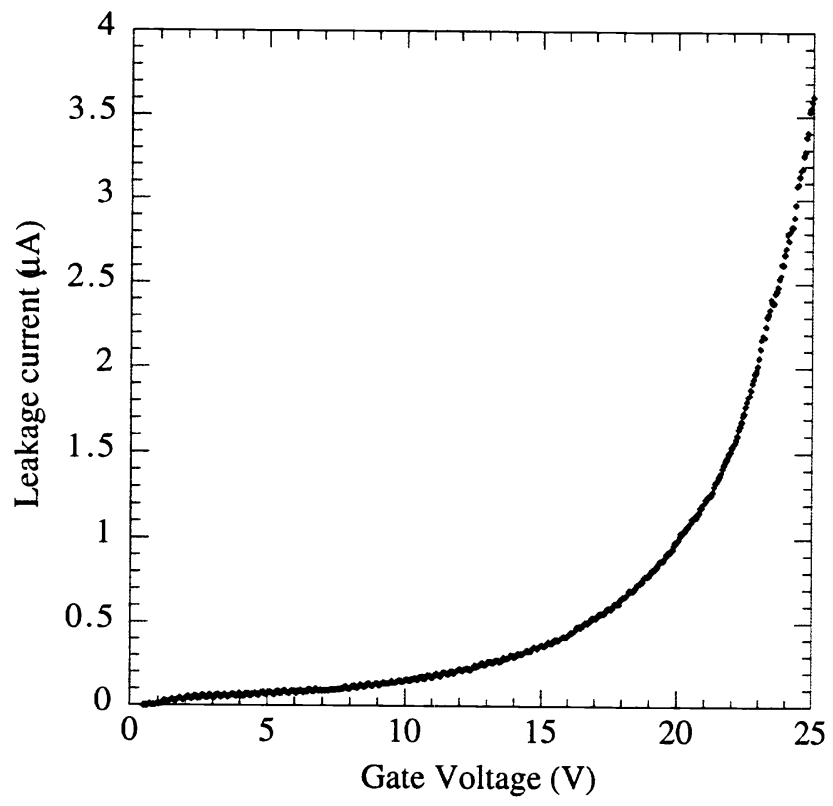

Figure 7. Reverse leakage current through Schottky contacts showing very small additional current contributed from gate contacts on a $25 \times 500 \mu \mathrm{m}$ laser. This corresponds to a leakage density of less than $32 \mathrm{~mA} / \mathrm{cm}^{2}$. Broad area laser threshold density for this material was approximately $260 \mathrm{~A} / \mathrm{cm}^{2}$

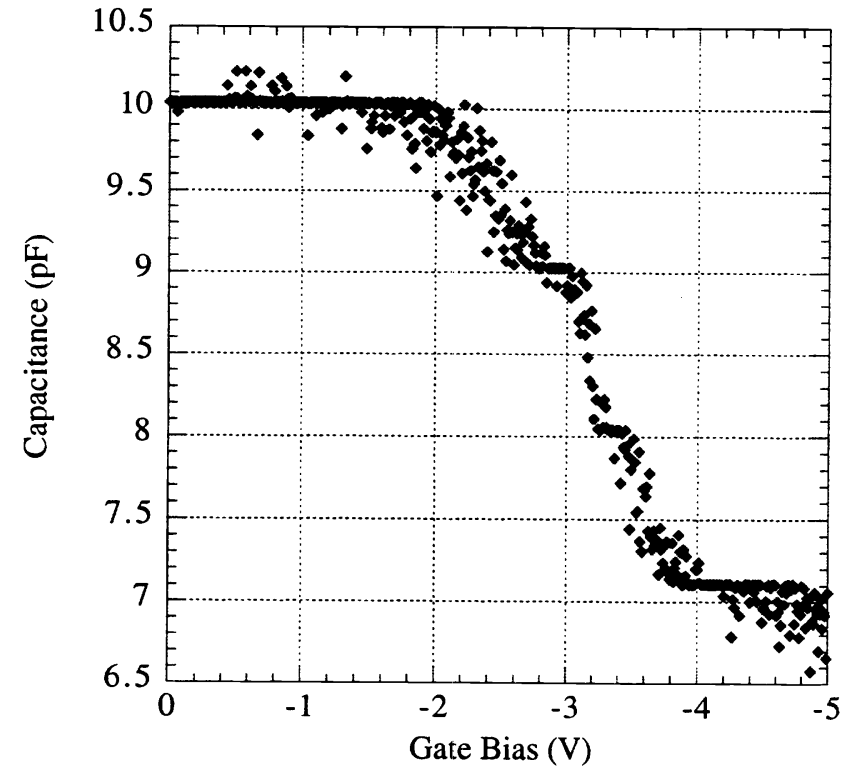

Figure 8. Capacitance plot for varying gate voltage for a $25 \mu m \times 600 \mu m$ laser

Typically, in Schottky and MIS devices, capacitance measurements can be used to estimate depletion layer thicknesses. Figure 8 shows a capacitance versus voltage plot for the gate contact of a typical laser device. Due to the complex geometry of this gate structure, an effective depletion width is not easily estimated. However, for an order of magnitude estimate we can use a simple parallel plate capacitor approximation to calculate the change in 


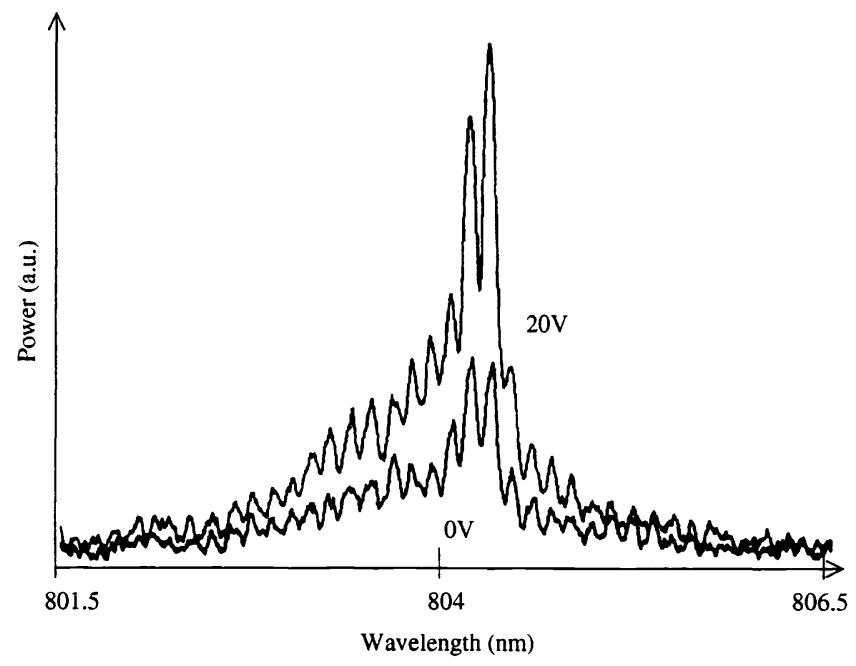

Figure 9. Lasing spectrum of depletion controlled laser at different gate voltages showing no measurable shift in the mode wavelengths. The laser was $60 \times 600 \mu m$

depletion width as

$$
\Delta w=\epsilon A\left(\frac{1}{C(V)}-\frac{1}{C(0)}\right)
$$

where $\Delta w$ is the change in the depletion width (and also the change in the effective laser width), and $C(V)$ is the capacitance as a function of applied bias. It is assumed that at the zero bias point, $C(0)$, the depletion layer is negligible compared to the ridge width. For the device shown in figure 8 this gives an estimate of the depletion width change of approximately $0.2 \mu \mathrm{m}$ which is consistent with the estimated change in the effective active width estimated from threshold current changes shown in figure 6 . It should be noted that although capacitance measurements and threshold current data presented here are from lasers of different width, the capacitance measurement will depend mostly on the Schottky contact size which was the same in both cases and not the laser width.

The lasing spectrum for a laser $60 \mu m$ wide and approximately $600 \mu m$ long is given in figure 9 for gate voltages of $0 \mathrm{~V}$ and $20 \mathrm{~V}$, pumped slightly above threshold. This laser is multimode and the increase in output power is clearly seen with no measurable shift in the lasing wavelengths. This indicates that the effect of the depletion region on the round trip cavity phase shift is small enough so that the modes seen in the lasing spectrum are unaffected to the resolution of the measurement system, and confirms that the electrostatic gates only contribute to the carrier distribution within the laser cavity. Figure 10 shows the variation in the integrated power with change in the gate voltage at constant current pumping slightly above the lasing threshold for the same $60 \times 600 \mu m$ device. It is clear from figure 10 that significant change in the optical power from the laser is achieved with constant current pumping simply by varying the gate voltage. The output power was increased by a factor of approximately 1.5 for a gate voltage change of $0 \mathrm{~V}$ to $25 \mathrm{~V}$. The leakage current through the gate contact for this device is shown in figure 7 and is seen to remain below $4 \mu A$ for the bias region of interest. Thus the gate leakage current cannot account for the significant optical power increase. Examination of the near field distribution in this case showed a shift in the optical mode. The gate bias was applied from only one side of the waveguide and the optical mode was seen to shift away from the gate contact with increasing bias voltage. The current-voltage characteristics of the laser PN-junction is shown in figure 11. The laser is pumped through the low doped cladding layer used for the gate contact.

\section{CONCLUSION}

We demonstrate electrostatic gating as a new method for reproducibly controlling threshold currents in semiconductor laser diodes. This approach for reducing carrier diffusion and thereby threshold currents in lasers relies on electrostatic depletion of carriers away from the edges rim of the laser diodes. The approach we chose for gating was to use a reversebiased metal-semiconductor Schottky diode or a MOS-type electrostatic depletion gate. Thus, our optoelectronic 


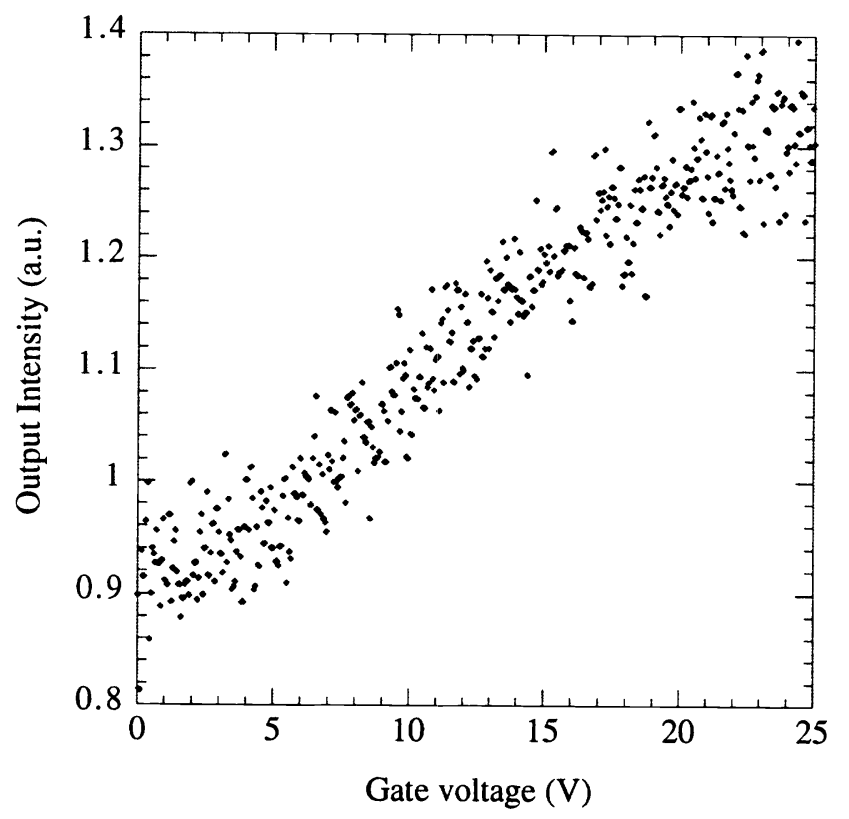

Figure 10. Increasing power with gate voltage above threshold for a $600 \times 60 \mu m$ laser

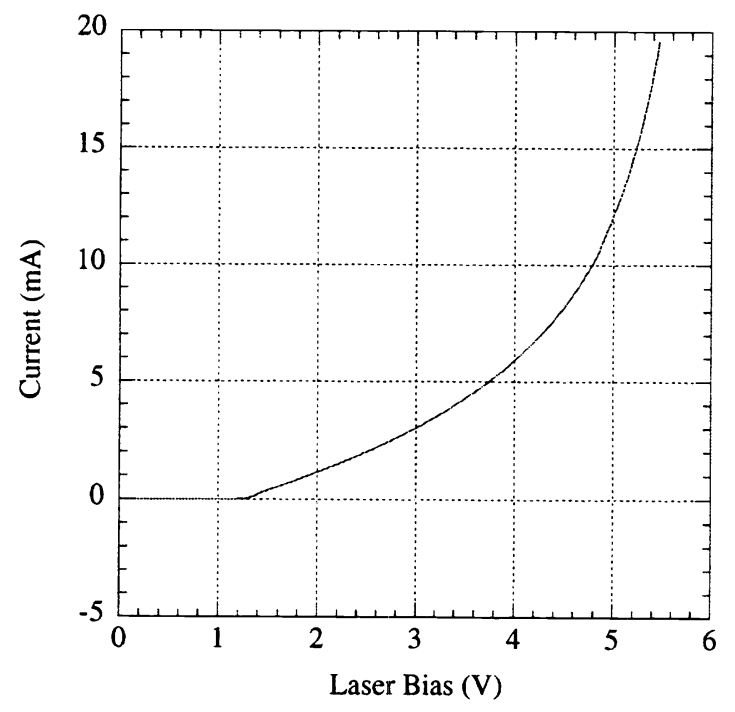

Figure 11. Current-voltage characteristics of the laser PN-junction with $2 \mu m$ low-doped layer in the p-doped cladding for the gate contacts 
device which in many ways resembles a lateral metal-semiconductor field effect transistor (MESFET). We have essentially, within the optical waveguide region, integrated a MESFET on top of the PIN junction of the laser diode. Electrostatic depletion also allows the formation of a separate carrier confinement structure in the lateral direction of a laser cavity, which is especially important for vertical cavity surface emitting laser diodes (VCSELs). The optical and carrier confinement in this device are thus separated, resulting in laser performance gains similar to that of epitaxially grown carrier confinement structures commonly used in efficient edge-emitting laser diodes. In addition to being able to electrostatically reduce or tune the active volume and thereby the threshold current of a laser, the gate also allows the electrostatic modulation of the laser power with constant current pumping above the lasing threshold. Although it could be argued that the additional contacts necessary for the inclusion of a third terminal into a 2-terminal diode structure result in more demanding fabrication and chip real-estate, we have developed and demonstrated here self-aligned devices which are simple to fabricate even for small laser diodes widths.

\section{ACKNOWLEDGEMENTS}

The authors gratefully acknowledge the funding support for this project from the Advanced Research Projects Agency and the Office of Naval Research. R.K.Lee and O.J.Painter also receive support from the Natural Science and Engineering Research Council of Canada.

\section{REFERENCES}

1. H. Zhao, M. M. ad P.D. Dapkus, K. Uppal, Y. Cheng, and G. Yang, "Submilliampere threshold current In$\mathrm{GaAs} / \mathrm{GaAs} / \mathrm{AlGaAs}$ lasers and laser arrays grown on nonplanar substrates," J. Select. Topics Quantum Electron. 1, pp. 196-202, 1995.

2. Y. Sin, H. Horikawa, Y. Matsui, and T. Kamijoh, "Ultralow laser threshold and high speed InGaAs-GaAs-InGaP buried heterostructure strained quantum well lasers for optical interconnects," Elect. Lett. 29, pp. 873-874, 1993.

3. Y. Cheng, P. Dapkus, M. MacDougal, and G. Yang, "Lasing characteristics of high-performance narrow-stripe InGaAs-GaAs quantum-well lasers confined by AlAs native oxide," IEEE Photon. Tech. Lett. 8, pp. 176-179, 1996.

4. M. Amann, "New stripe-geometry laser with simplified fabrication process," Elect. Lett. 15, pp. 441-442, 1979.

5. H. Temkin, A. Chin, and B. Dutt, "Schottky barrier restricted GaAlAs laser," Elect. Lett. 18, pp. 701-703, 1982.

6. J. Dallesasse, N. Holonyak, A. Sugg, T. Richard, and N. El-Zein, "Hydrolyzation oxidation of $A l_{x} G a_{1-x} A s-$ AlAs - GaAs quantum well heterostructures and superlattices," App. Phys. Lett. 57(26), pp. 2844-2846, 1990.

7. E. Chen, N. Holonyak, and S. Maranowski, " $A l_{x} G a_{1-x} A s-G a A s$ metal-oxide semiconductor field effect transistors formed by lateral water vapor oxidation of AlAs," App. Phys. Lett. 66(20), pp. 2688-2690, 1995.

8. A. Chin, C. Zipfel, B. Dutt, M. DiGiuseppe, K. Bauers, and D. Roccasecca, "New restricted contact LEDs using a Schottky barrier," Jap. J. App. Phys. 20, pp. 1487-1491, 1981.

9. A. Chin, C. Zipfel, and B. Dutt, "The reliability of Schottky barrier restricted GaAs/GaAlAs LEDs," Jap. J. App. Phys. 21, pp. 1308-1312, 1982. 